\title{
INFRARED OBSERVATION DURING THORACOSCOPIC SURGERY FOR BULLOUS DISEASE
}

\author{
Takashi Suzuki, MD, Akihiko Kitami, MD, Shuichi Suzuki, MD, Yoshito Kamio, MD, Yasunori Hida, MD, and \\ Goichi Hori, MD, Yokohama, Japan
}

In thoracoscopic operations for bullous diseases involving a giant bulla and a spontaneous pneumothorax, it is sometimes difficult to determine the margin of pulmonary resection and to find a tiny bleb. A new instrument to examine the pleural or subpleural structure more precisely is necessary. In the field of ophthalmology, the use of nearinfrared rays in in vivo observations has established their usefulness because of their permeability to a vital tissue. Therefore the observation of the pleural lesion under nearinfrared rays is thought to be effective for imaging pleural and subpleural lesions. We used a system of infrared observation for detecting bleb or bullous lesions in thoracoscopic operations.

Methods and patients. The infrared observation system used an A5288A thoracoscope (Olympus Co) that was 10 $\mathrm{mm}$ in diameter, with a visual field of $30^{\circ}$. The lens was coated to enable infrared ray penetration. The light source was a CLV-U20 (Olympus Co) adjusted to generate an 805-nm infrared ray. In addition to this infrared observation system, the typical white-light view was also used.

From the Department of Thoracic and Cardiovascular Surgery, Showa University Fujigaoka Hospital, Yokohama, Japan.

The infrared observation system used in this study was borrowed from Olympus Co.

Received for publication July 15, 1999; accepted for publication Aug 17, 1999.

Address for reprints: Takashi Suzuki, MD, Department of Thoracic and Cardiovascular Surgery, Showa University Fujigaoka Hospital, 1-30 Fujigaoka, Aoba-ku, Yokoham, Japan.

J Thorac Cardiovasc Surg 2000;119:182-4

Copyright $@ 2000$ by Mosby, Inc.

$0022-5223 / 2000 \$ 12.00+0 \quad \mathbf{1 2 / 5 4 / 1 0 2 2 9 4}$
Subjects consisted of 15 patients with spontaneous pneumothorax, 3 sustaining giant bullae, and 1 with lung cancer as a control subject. All the operations on patients, except for the patient with lung cancer, were performed during thoracoscopy. The patient with lung cancer in the right lower lobe first underwent thoracoscopic observation of all lobes; thereafter, typical thoracotomy was done for lower lobectomy. The normal lung tissue of the lower lobe corresponding to the thoracoscopic observation, apart from lung cancer, was submitted to histologic examination after lobectomy. The resected specimens of bullous diseases were evaluated macroscopically and microscopically.

Results. The normal lungs were observed as a white organ containing numerous tiny vesicles beneath the pleura on the infrared image, looking like white snow. The tiny vesicles corresponded to alveoli located in the subpleural area, and their presence denoted a characteristic feature of the normal lung structure (Fig 1). Because atelectasis only showed the gray lung, inflation by an anesthetist was necessary. Black flecks of pleura were indicative of anthracosis caused by cigarette smoking or urban living, but they were not pathologic (Fig 1). Bullae and blebs were observed as a gray shimmering membrane protruding into the thoracic cavity concomitant with inflation of the lung (Figs 1 and 2). Particularly, the finding of a small gray translucent membrane was beneficial to reveal a tiny bleb (Fig 2). The emphysematous lung did not have the white snow structure under the pleura; rather it showed a finding like a gray balloon. These findings were useful to determine the resection line of the giant emphysematous bulla.

The resection of the lesion was performed primarily under the white-light system; the infrared system occasionally supported the typical system.

The disadvantage of the infrared observation was the 


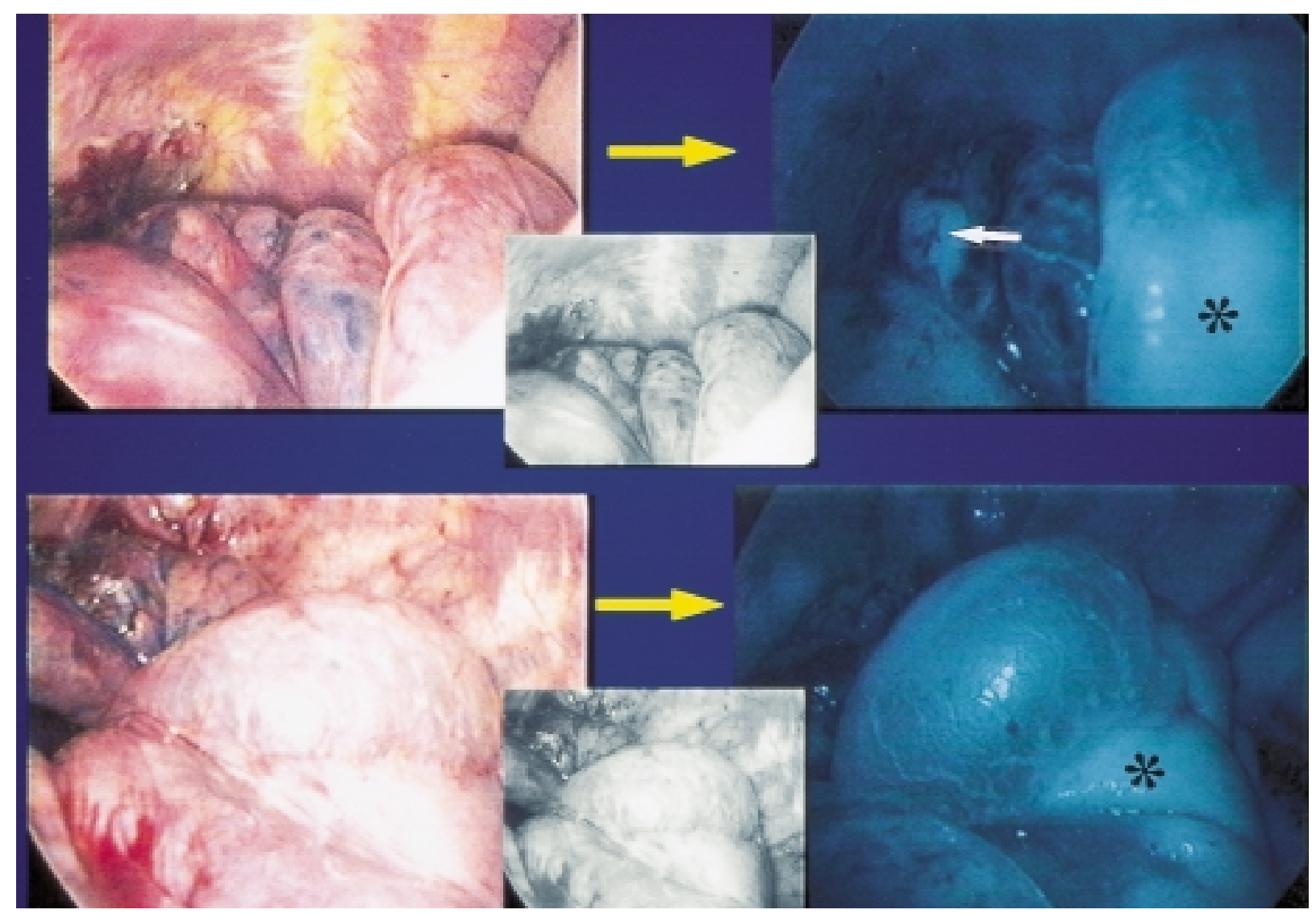

Fig 1. Left, Typical white light observation; right, infrared observation. Two yellow arrows indicate the corresponding images. Middle, Gray-scale image converted from the typical white-light image. Bullae were observed as gray shimmering membranes protruding into the thoracic cavity in the infrared observation. The normal pleura and lung surrounding the bulla were observed as a white structure, including small vesicles similar to white snow in the infrared observation (*). Black flecks of pleura were indicative of anthracosis (white arrow). The middle-gray images were only converted from the color images of the white-light observation; it could not reveal the pleural or subpleural lesions. The difference between the middle and right images was clear.

inability to detect bleeding because blood did not present a red color. However, no operative complications and no operative deaths occurred.

Discussion. We sometimes encounter a case of bullous disease, including spontaneous pneumothorax, the responsible bleb or margin of emphysematous lesion of which is difficult to detect. In accord with our experience, Inderbitzi and colleagues ${ }^{1}$ reported that endoscopic examination failed to reveal lung lesions in $5.1 \%$ of their patients with spontaneous pneumothorax. Naunheim and colleagues ${ }^{2}$ could not identify blebs or bullae in $9 \%$ of the 113 patients; they also reported that failure of identification brought a high risk of recurrence. On the other hand, as a trial for detecting a responsible bleb, Melvin and colleagues ${ }^{3}$ reported a marking method with an intraoperative bronchoscope in which methylene blue was instilled into the bronchi. Thereafter the lesion out of which dye flowed could be detected under the thoracoscope. The infrared observation system that we used was useful to detect fine pleural lesions without the use of other bothersome modalities, such as bronchoscopy during surgery.

Fine blebs could be detected clearly by this method; however, it is unclear whether the prophylactic resection of tiny blebs contributes to reducing the recurrence of spontaneous pneumothorax. We usually try to resect tiny blebs as much as possible, but for patients whose lung volume should be preserved, we do not resect all the blebs or bullae without a finding of air leakage. The evolution of tiny blebs is an issue for the future.

On the other hand, in volume reduction surgery it is sometimes difficult to determine the target area and cutting line under thoracoscopy. The infrared observation system is also thought to be useful in evaluating the emphysematous lung to be resected. Although we do not have enough experience to comment on the usefulness of the system in volume reduction surgery under thoracoscopy, the infrared observation system had an advantage in the thoracoscopic surgery of giant bullae. 


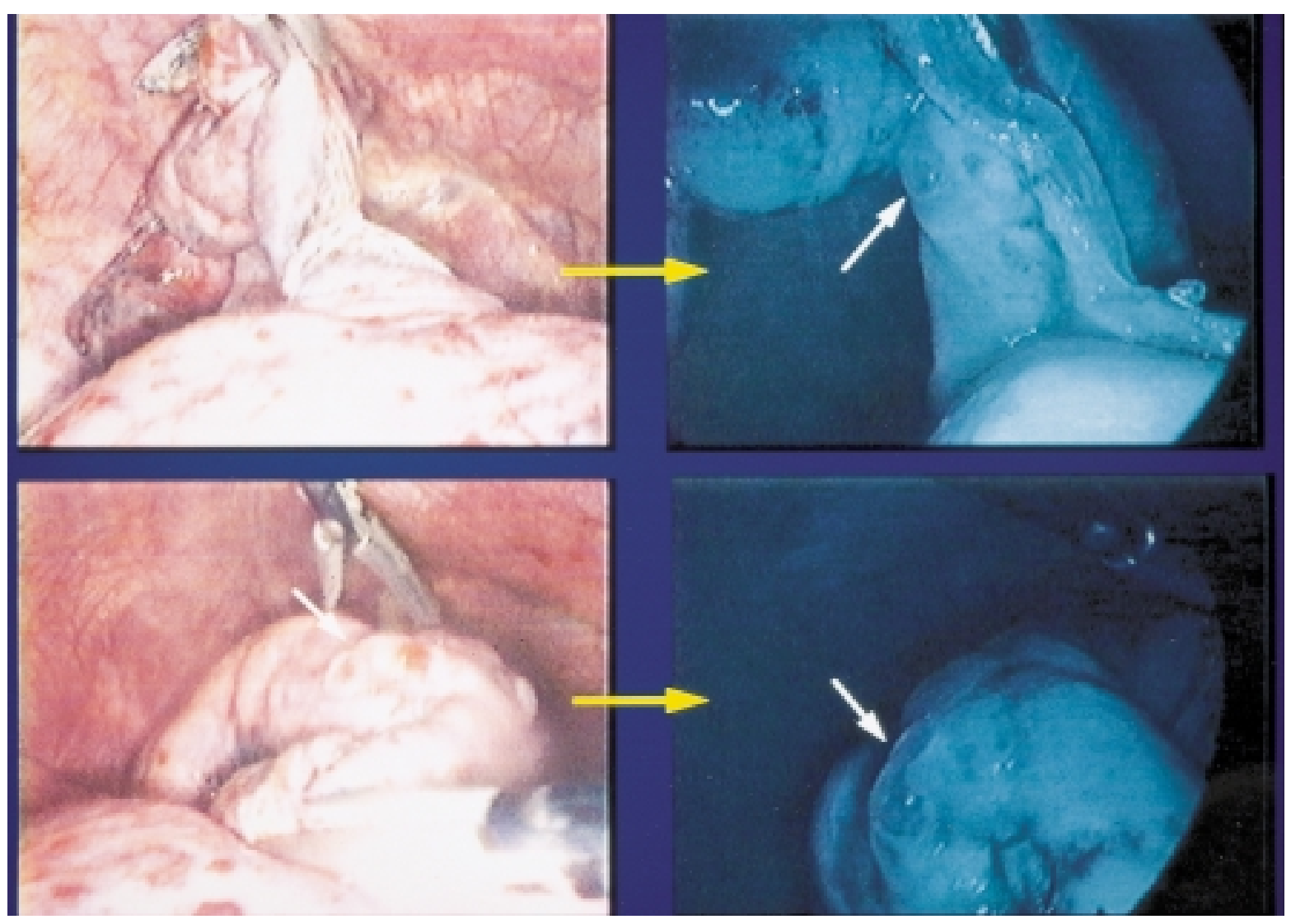

Fig 2. Left, Typical white-light observation; right, infrared observation. Two yellow arrows indicate the corresponding images. The lesions were cut by using an endoscopic linear stapler. During the thoracoscopic resection, the location of the lesions could be confirmed more accurately by the infrared observation. Small white arrows indicate tiny blebs; however, the left upper typical image did not reveal the bleb that was revealed by infrared observation.

\section{REFERENCES}

1. Inderbitizi RGC, Leiser A, Furrer M, Althaus U. Three years' experience in video-assisted thoracic surgery (VATS) for spontaneous pneumothorax. J Thorac Cardiovasc Surg 1994;107:1410-5.

2. Naunheim KS, Mack MJ, Hazelrigg SR, et al. Safety and effi- cacy of video-assisted thoracic surgical techniques for the treatment of spontaneous pneumothorax. J Thorac Cardiovasc Surg 1995;109:1198-203

3. Melvin WS, Krasna MJ, McLaughlin JS. Thorascopic management of spontaneous pneumothorax. Chest 1992;102:1877-79. 